\title{
Management of Post-Traumatic Posterior Rectal Pouch Through a Posterior Approach
}

\author{
Sameh Hany Emile, MD*; Mohammad Fathy, MBBCh; Waleed Thabet, MD \\ Colorectal Surgery Unit, General Surgery Department, Mansoura Faculty of Medicine, Mansoura University Hospitals, \\ Mansoura University, Mansoura City, Egypt
}

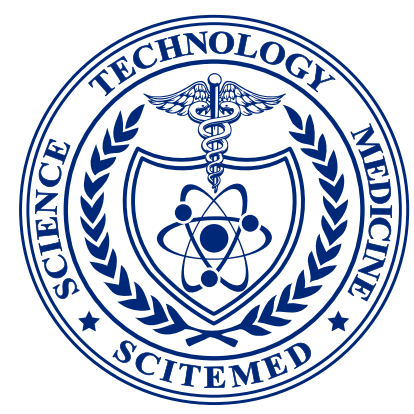

\begin{abstract}
Traumatic rectal injury is caused by a variety of insults and can be associated with high morbidity. Fecal diversion, rectal washout, and presacral drainage are considered the essential components of treatment of traumatic rectal injuries. A 25-year-old male patient sustained a gunshot injury to the pelvis that resulted in a posterior rectal perforation communicating with the sacrococcygeal region. The patient was initially managed with diverting sigmoid colostomy and the recto-cutaneous fistula was left to heal by secondary intention. Two years later, the patient came for the closure of colostomy. Rectal contrast study showed a large pouch posterior to and communicating with the rectum. A posterior approach was used to deal with the posterior rectal pouch, which was a chronic abscess cavity encapsulated with a layer of fibrous tissue. The pouch was dissected from the surrounding structures and excised at its neck. The resultant rectal defect was repaired by interrupted full-thickness sutures and was reinforced by approximation of the overlying fascia. The postoperative course was uneventful. The posterior approach enabled complete excision of a well-defined chronic abscess cavity that was communicating with the rectal lumen along with full-thickness repair of the rectal defect. An important privilege of pouch excision through the posterior approach was preserving the rectal reservoir and sensation that would have been compromised if proctectomy with coloanal anastomosis was alternatively performed.
\end{abstract}

\section{Introduction}

Penetrating rectal injuries are considered one of the important challenges for colorectal surgeons. These injuries are usually inflicted by penetrating stab, gunshot, or pelvic fracture secondary to a motor vehicle accident or falling from a height.

Rectal injuries are clinically graded, according to the American Association for the Surgery of Trauma (AAST), into five distinct grades: grade I (contusion or partial thickness laceration), grade II (laceration involving less than half of the circumference), grade III (laceration of more than half of the circumference), grade IV (full-thickness laceration with perineal extension), and grade $\mathrm{V}$ (devascularized segment) [1].

The decision-making strategy for the initial treatment of rectal injuries relies on a number of factors that include the anatomic site and cause of rectal injury, physiologic condition of the patient, and antibiotic use [2,3]. It has been recognized that the rectal injuries above the peritoneal reflection are readily accessible and amenable to treatment as for colonic injuries, and hence they are classically treated with primary repair with or without proximal diversion [4]; on the other hand, the extraperitoneal rectal injuries are usually more difficult to access and direct repair is usually impossible [5].

The Eastern Association for the Surgery of Trauma (EAST) has devised a set of evidence-based recommendations for the management of penetrating extraperitoneal rectal injuries [6]. The practice guidelines addressed three main questions about the indications for presacral drainage, distal rectal washout, and proximal fecal diversion.

Although presacral drainage and rectal washout were once considered the basic principles for the management of extraperitoneal rectal injuries [7], the EAST guidelines recommended against their routine use for the initial management of extraperitoneal rectal perforation. After analyzing 17 studies, it was found that omitting presacral drainage resulted in a $40 \%$ reduction of the overall infectious complication rate with comparable mortality rates of drainage and non-drainage groups. Similarly, upon analysis of 13 studies, the mortality and infectious complications rates in patients who had or did not have distal rectal washout were similar, $(0.99 \%$ vs. $1.37 \%)$ and $(9.9 \%$ vs. $10.3 \%)$, respectively.
Furthermore, a recent multi-institutional study [8] found that the presacral drainage and rectal washout were independently associated with a three-fold increase in abdominal complications, recommending to avoid these measures in the management of extraperitoneal rectal injuries. While the role of presacral drainage and distal rectal washout in the setting of civilian rectal injury is debatable, they still have a place in the battlefield or other high-energy transfer rectal injuries in order to decrease septic complications, as Barkley et al. [5] implied.

The role of fecal diversion in the management of rectal injuries remains controversial. The EAST practice guidelines [7] endorsed proximal diversion as a mainstay in the treatment of extraperitoneal rectal injuries after reviewing the outcome of 14 studies and concluding that using proximal diversion served to achieve no mortality and reduced the infectious complication rate by $50 \%$ compared to the non-diversion group. Conversely, other investigators [2] concluded that "colostomy is no longer mandatory for all rectal injuries", implying that proximal diversion is mainly indicated for high-grade destructive injuries, presence of fecal contamination, delayed presentation, and anal sphincter injury.

In addition to the initial treatment, the management of rectal injuries also entails treatment of subsequent complications and sequelae of trauma. The most common complications of rectal trauma are septic complications including wound infection, pelvic abscess, or generalized sepsis. Full-thickness rectal injuries are associated with a three times higher rate of complications, compared to partial thickness injuries [5]. The present report describes the management of a delayed complication of penetrating extra-peritoneal rectal injury that is presacral abscess cavity communicating with the rectal lumen creating a pseudo-pouch.

\section{Case Report}

In this report, we present the case of an otherwise healthy 25-year-old male patient who sustained a gunshot injury to the pelvis, four years ago. A high-velocity missile made its way entering from the left iliac fossa and exiting through the sacrococcygeal region. The patient was resuscitated in the emergency department of another health care facility, and then underwent an urgent exploratory laparotomy that revealed a single perforation of the terminal ileum and another larger perforation in the pos- 
terior rectal wall. Resection of the injured ileal loop with re-anastomosis of the bowel ends was done, whereas no attempt of repair of the rectal injury was made, and a diverting sigmoid colostomy was constructed. The resultant fistula between the posterior rectal wall and the skin of the sacrococcygeal region was left to heal by secondary intention, which took around 24 months to accomplish.

The patient was referred to our colorectal surgery unit for the possibility of reversal of colostomy. On assessment of the general condition, the patient had no history of medical comorbidities such as diabetes mellitus, hypertension, bronchial asthma, or cardiac disease. The patient was not on chronic steroid or immunosuppressive therapy. The routine laboratory investigations ordered for the patient revealed no abnormalities. Rectal contrast study was performed and showed an abscess cavity connecting to the posterior rectal wall through a large ostium measuring about $4.5 \mathrm{~cm}$ in diameter. The barium-filled pouch was located anterior to the sacrum with no visible extravasation of the dye beyond its boundaries (Figure 1A).

Examination under anesthesia revealed a $5 \mathrm{~cm}$ defect in the posterior rectal wall, around two inches above the anal verge. This rectal defect was connected with a large pouch filled with pus and granulation tissue, with the upper part of the pouch lying behind the sacrum.

The patient was scheduled for excision of the pouch with simultaneous repair of the rectal wall through a posterior approach. Mechanical and chemical bowel preparations were conducted preoperatively according to the standard protocol. A single dose of prophylactic antibiotics (1 $\mathrm{gm}$ of $3^{\text {rd }}$ generation cephalosporins and $500 \mathrm{mg}$ of metronidazole) was given on induction. The patient was placed in a prone jack-knife position and the procedure was performed by a consultant of colorectal surgery under general anesthesia.

A vertical midline incision was made over the sacrococcygeal region with its distal end around one inch above the anal verge. The subcutaneous tissue and fascia were divided longitudinally with electrocautery until the rectal pouch was identified. A small incision was made at the middle of the pouch (Figure 1B) to permit admission of the index finger into its cavity to delineate its boundaries and facilitate its dissection from the surrounding structures.

Using electrocautery, the pouch was dissected superiorly and anteriorly from the sacrum, bilaterally from ischial bones, and inferiorly from the puborectalis muscle. The dissection was continued till reaching the point of connection between the pouch and the rectal wall, and then the pouch was completely excised leaving a $5 \times 3 \mathrm{~cm}$ defect in the posterior rectal wall, through which the rectal mucosa was clearly visible (Figure 1C). The edges of the rectal defect were trimmed using sharp scissors, dissected, and then approximated longitudinally using full-thickness, interrupted polyglactin 2-0 sutures (Figure 1D). Afterwards, the fascia was closed longitudinally with interrupted sutures (Figure $1 \mathrm{E}$ ), a negative suction drain was placed, and the skin was closed with interrupted polypropylene 2-0 sutures (Figure 1F).

The patient was monitored in the surgical ward for five days. One gram of $3^{\text {rd }}$ generation cephalosporin and $500 \mathrm{mg}$ metronidazole were administered intravenously every 12 hours in the first 48 hours postoperatively. Analgesics (diclofenac sodium) were administered when appropriate. The volume of drainage in the suction drain was measured on daily basis. Overall, the postoperative course was uneventful after follow-up for two months with no recorded complications. A written informed consent for publishing this case report was obtained from the patient.

\section{Discussion}

Penetrating rectal injuries remain a challenging surgical problem despite the advances in imaging modalities and surgical techniques. The present report describes a young male patient with grade III injury of the extraperitoneal rectum secondary to a gunshot. Although the mechanism of injury that led to the small bowel and rectal perforation is not clear, we can assume that either it was due to a very steep trajectory of the bullet breaching only the posterior wall of the rectum or because of disintegration or fragmentation of the bullet inside the body.
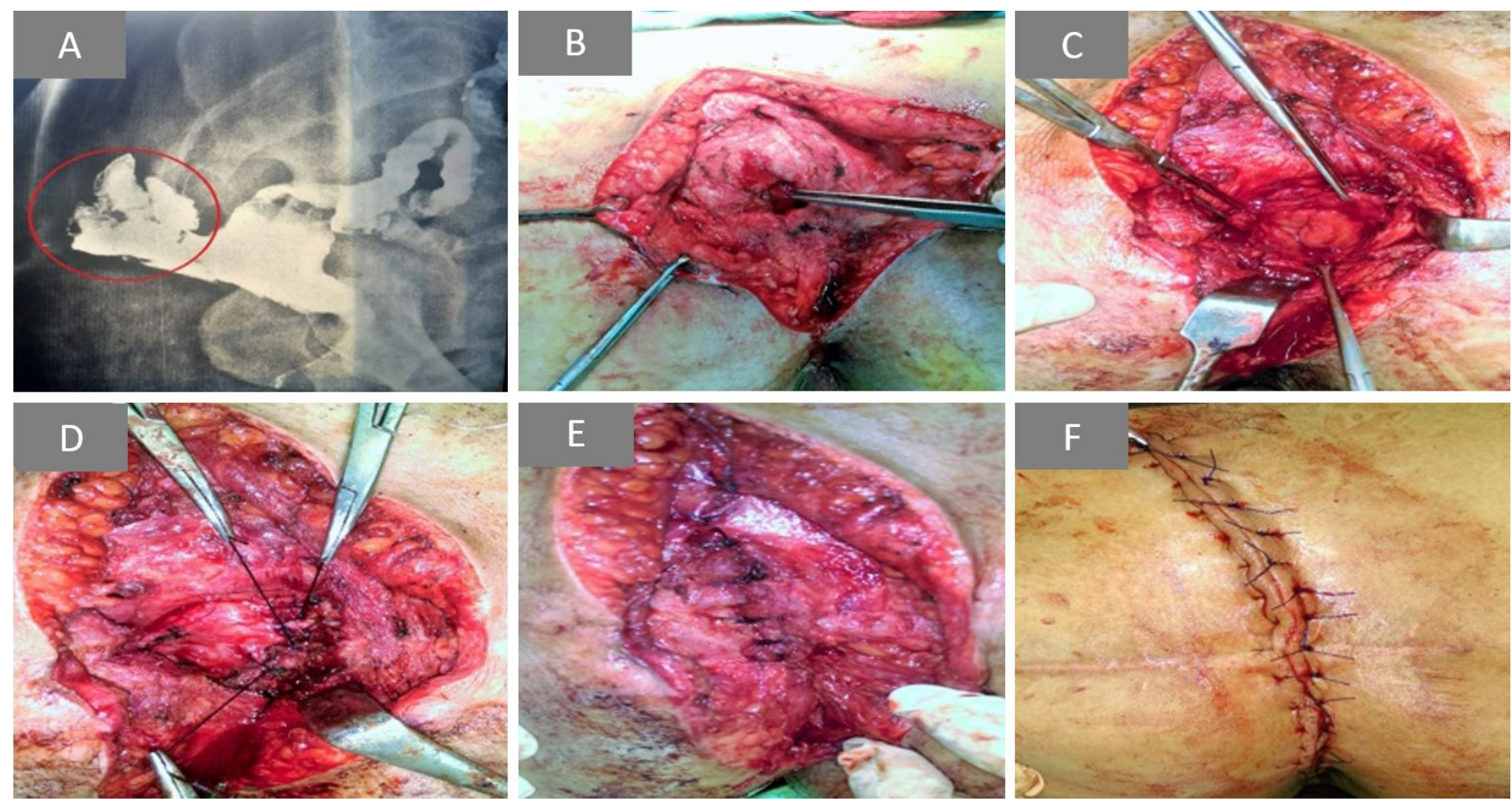

Figure 1. (A) Rectal contrast study demonstrating large pouch posterior to and communicating with the rectum. (B) Posterior rectal pouch with small incision made at its middle. (C) The resultant defect at the posterior rectal wall after pouch excision. (D) Closure of the rectal wall defect with interrupted full-thickness statures. (E) Approximation of the fascial layer over the repaired rectal defect. (F) Final view after skin closure. 
Some investigators $[2,9]$ advocated primary repair without proximal diversion for extraperitoneal rectal injuries; however, the patient of the current report was managed with diverting colostomy without conducting the primary repair. Perhaps, the site and high grade of rectal injury, in addition to the association with small bowel injury, precluded any attempt of primary repair of the rectal perforation.

As a consequence of the missile injury, the posterior rectal wall became communicating with the sacrococcygeal region forming a recto-cutaneous fistula. With antibiotic therapy and daily debridement and dressing, the external sacrococcygeal wound healed with granulation tissue that matured into fibrous tissue leaving an abscess cavity behind. The proximal diversion prevented further fecal contamination of this abscess cavity allowing it to become encompassed with a layer of fibrous tissue, and converting it to a pseudo-pouch connected to the rectum through the site of rectal perforation.

Rectal contrast studies are crucial before planning closure of diverting colostomy after rectal injuries. Water-soluble contrast agents, such as Gastrografin ${ }^{\circledR}$, are often used to detect leakage in the suspected rectal injury, whereas barium sulfate is contraindicated as it can result in devastating barium peritonitis. A single contrast technique is used to identify the site of perforation and the bowel preparation is usually not necessary [10]. It is worthy to note that although the rectal contrast agent leakage is a highly specific sign (96-98\%), it is also insensitive (42-46\%), and hence its absence cannot be used to assertively exclude rectal perforation [11].

In this case of this report, the contrast study revealed an important anomaly that is the posterior rectal pouch. Should the restoration of the continuity of the colon was undertaken without prior contrast study, the dormant rectal pouch would be contaminated with fecal matter and could turn into an active abscess with the possibility of perforation.

The present report addressed one of the delayed complications of traumatic rectal injury that is posterior rectal pseudo-pouch. Brown et al. [12] analyzed the complications of penetrating rectal injury and its surgical treatment and found that around $8 \%$ of the patients with rectal injury developed delayed complications in the form of intra-abdominal abscess, urinary incontinence, and hernia. Careful evaluation and follow-up of the patients who sustained rectal injury are mandatory to identify and properly treat any delayed complications of rectal injury or surgical intervention performed for its management.

Two options were available for the treatment of this low-lying posterior rectal pouch: either through a trans-abdominal approach and then performing a formal low anterior resection with coloanal anastomosis, or through a posterior approach and then excising the pouch at its neck with repair of the resultant rectal defect.

Extraperitoneal rectal injuries can be approached and repaired transabdominally during laparotomy; however, extensive mobilization of the rectum should be avoided. We did not prefer the transabdominal approach for two reasons: the first, we thought conducting a transabdominal low rectal resection would be difficult owing to the expected adhesions in the pelvis in addition to the morbidity of laparotomy in general; the second reason was to preserve the rectal reservoir function in this young patient. Since the rectal defect was two inches above the anal verge, a low coloanal anastomosis would have been performed, which might entail the risk of anastomotic leakage and functional disturbances as low anterior resection syndrome [13].

We opted to repair the rectal injury and the associated abscess cavity through a posterior approach (Kraske procedure) owing to their anatomic location. The Kraske approach provides adequate exposure of the presacral space, enabling the surgeon to visualize and deal with mid-rectal lesions efficiently [14]. Although the previous reports [15] discussed the utility of the posterior rectal approach in the treatment of various benign and malignant lesions, this report is the first to describe the treatment of delayed complications of traumatic rectal injury via the posterior approach. Indeed, the posterior approach enabled access to the posterior rectal pouch with the privilege of complete dissection of the pouch under direct vision. Upon complete dissection and excision of the pouch, the rectal mucosa was clearly seen bulging through the defect. Full-thickness repair of the rectal defect was feasible and the fascial closure provid- ed further reinforcement of the repair. The rectal contour was restored without the need for excising any segment of the rectum, thus preserving the rectal capacity and sensation.

Although the recent alternatives to surgical treatment, such as endo-sponge treatment or vacuum-assisted therapy [16], were also viable options in the cases of perirectal abscess cavities secondary to trauma or anastomotic leakage, the use of these modalities was not attempted since they were not commercially available in our country.

\section{Conclusion}

Proximal fecal diversion is the treatment of choice in the cases of extra-peritoneal rectal injuries that are not amenable for primary repair due to their distant location or high grade. The posterior approach enables complete excision of well-defined abscess cavities that communicate with the rectal lumen with full-thickness repair of the rectal defect. An important privilege of pouch excision through the posterior approach was the preservation of rectal reservoir and the sensation that would have been compromised if proctectomy with coloanal anastomosis was alternatively performed.

\section{Article Information}

*Correspondence: Sameh Hany Emile, MD. General Surgery Department, Faculty of Medicine, Mansoura University Hospitals, El Gomhouria St, Mit Khamis WA Kafr Al Mougi, Mansoura, Dakahlia Governorate, Egypt. Email: sameh200@hotmail.com

Received: Jul. 17, 2018; Accepted: Aug. 09, 2018; Published: Sep. 19, 2018

DOI: 10.24983/scitemed.acri.2018.00081

Copyright (c) 2018 The Author(s). This is an open-access article distributed under the terms of the Creative Commons Attribution 4.0 International License (CC-BY).

\section{Funding: None}

\section{Conflict of Interest: None}

\section{Keywords}

Posterior approach; post-traumatic; pouch; rectal injury.

\section{References}

1. Moore EE, Cogbill TH, Malangoni MA, et al. Organ injury scaling, II: Pancreas, duodenum, small bowel, colon, and rectum.J Trauma 1990;30(11):1427-1429.

2. Ulger BV, Turkoglu A, Oguz A, Uslukaya O, Aliosmanoglu I, Gul M. Is ostomy still mandatory in rectal injuries? Int Surg 2013;98(4):300303.

3. Weinberg J, Fabian T, Magnotti LJ, et al. Penetrating rectal trauma: management by anatomic distinction improves outcome. J Traum 2006; 60(3):508-513; discussion 513-514.

4. Fouda $\mathrm{E}$, Emile $\mathrm{S}$, Elfeki $\mathrm{H}$, et al. Indications for and outcome of primary repair compared with faecal diversion in the management of traumatic colon injury. Colorectal Dis 2016;18(8):0283O291.

5. Barkley S, Khan M, Garner J. Rectal trauma in adults. Trauma 2013;15(1):3-15.

6. Bosarge PL1, Como Jl, Fox N, et al. Management of penetrating extraperitoneal rectal injuries: an Eastern Association for the Surgery of Trauma practice management guideline. J Trauma Acute Care Surg $2016 ; 80(3): 546-551$.

7. Choi WJ. Management of colorectal trauma.J Korean Soc Coloproctol 2011;27(4):166-172.

8. Brown CVR, Teixeira PG, Furay E, et al. Contemporary management of rectal injuries at Level I trauma centers: The results of an 


\section{CASE REPORT}

American Association for the Surgery of Trauma multi-institutional study. J Trauma Acute Care Surg 2018;84(2):225-233.

9. Levine JH, Longo WE, Pruitt C, Mazuski JE, Shapiro MJ, Durham RM. Management of selected rectal injuries by primary repair. $A m$ J Surg 1996 ;172(5):575-578; discussion 578-579.

10. DeStigter KK, Lefebvre SD. Imaging colorectal trauma. Semin CoIon Rectal Surg 2004;15:80-89.

11. Dreizin $D$, Boscak AR, Anstadt MJ, et al. penetrating colorectal injuries: diagnostic performance of multidetector CT with trajectography. Radiology 2016;281(3):749-762.

12. Brown SR, Swisher JP, Hofmann LJ, Coviello LC, Davis KG. Surgical management and associated complications of penetrating rectal injuries sustained in Iraq and Afghanistan. Mil Med 2013;178(11):1213-1217.
13. Keane C, Wells C, O'Grady G, Bissett IP. Defining low anterior resection syndrome: a systematic review of the literature. Colorectal Dis 2017;19(8):713-722.

14. Onaitis M, Ludwig K, Perez-Tamayo A, et al. The Kraske procedure: a critical analysis of a surgical approach for mid-rectal lesions. J Surg Oncol 2006;94(3):194-202.

15. Westbrook KC, Lang NP, Broadwater JR, Thompson BW. Posterior surgical approaches to the rectum. Ann Surg 1982;195(6): 677685.

16. Riss S, Stift A, Meier M, Haiden E, Grünberger T, Bergmann M. Endo-sponge assisted treatment of anastomotic leakage following colorectal surgery. Colorectal Dis 2010;12(7 Online):e104-e108. 